\title{
Adaptations in mitochondrial function parallel, but fail to rescue, the transition to severe hyperglycemia and hyperinsulinemia: a study in Zucker diabetic fatty rats.
}

Citation for published version (APA):

Lenaers, E., Feyter, H. M., Hoeks, J., Schrauwen, P., Schaart, G., Nabben, M., Nicolay, K., Prompers, J. J., \& Hesselink, M. K. (2010). Adaptations in mitochondrial function parallel, but fail to rescue, the transition to severe hyperglycemia and hyperinsulinemia: a study in Zucker diabetic fatty rats. Obesity, 18(6), 1100-1107. https://doi.org/10.1038/oby.2009.372

Document status and date:

Published: 01/01/2010

DOI:

10.1038/oby.2009.372

Document Version:

Publisher's PDF, also known as Version of record

Document license:

Taverne

Please check the document version of this publication:

- A submitted manuscript is the version of the article upon submission and before peer-review. There can be important differences between the submitted version and the official published version of record.

People interested in the research are advised to contact the author for the final version of the publication, or visit the DOI to the publisher's website.

- The final author version and the galley proof are versions of the publication after peer review.

- The final published version features the final layout of the paper including the volume, issue and page numbers.

Link to publication

\footnotetext{
General rights rights.

- You may freely distribute the URL identifying the publication in the public portal. please follow below link for the End User Agreement:

www.umlib.nl/taverne-license

Take down policy

If you believe that this document breaches copyright please contact us at:

repository@maastrichtuniversity.nl

providing details and we will investigate your claim.
}

Copyright and moral rights for the publications made accessible in the public portal are retained by the authors and/or other copyright owners and it is a condition of accessing publications that users recognise and abide by the legal requirements associated with these

- Users may download and print one copy of any publication from the public portal for the purpose of private study or research.

- You may not further distribute the material or use it for any profit-making activity or commercial gain

If the publication is distributed under the terms of Article $25 \mathrm{fa}$ of the Dutch Copyright Act, indicated by the "Taverne" license above, 


\title{
Adaptations in Mitochondrial Function Parallel, but Fail to Rescue, the Transition to Severe Hyperglycemia and Hyperinsulinemia: A Study in Zucker Diabetic Fatty Rats
}

\author{
Ellen Lenaers ${ }^{1,2}$, Henk M. De Feyter ${ }^{3}$, Joris Hoeks ${ }^{1,4}$, Patrick Schrauwen ${ }^{1,4}$, Gert Schaart ${ }^{1,2}$, \\ Miranda Nabben ${ }^{1,4}$, Klaas Nicolay ${ }^{3}$, Jeanine J. Prompers ${ }^{3}$ and Matthijs K.C. Hesselink ${ }^{1,2}$
}

Cross-sectional human studies have associated mitochondrial dysfunction to type 2 diabetes. We chose Zucker diabetic fatty (ZDF) rats as a model of progressive insulin resistance to examine whether intrinsic mitochondrial defects are required for development of type 2 diabetes. Muscle mitochondrial function was examined in 6-, 12-, and 19-week-old ZDF ( $\mathrm{fa} / \mathrm{fa}$ ) and fa/+ control rats ( $n=8-10$ per group) using respirometry with pyruvate, glutamate, and palmitoyl-CoA as substrates. Six-week-old normoglycemic-hyperinsulinemic fa/fa rats had reduced mitochondrial fat oxidative capacity. Adenosine diphosphate (ADP)-driven state 3 and carbonyl cyanide p-trifluoromethoxyphenylhydrazone (FCCP)-stimulated state uncoupled (state $u$ ) respiration on palmitoyl-CoA were lower compared to controls ( $62.3 \pm 9.5$ vs. $119.1 \pm 13.8$ and $87.8 \pm 13.3$ vs. $141.9 \pm 14.3 \mathrm{nmol} \mathrm{O} / \mathrm{mg} / \mathrm{min}$.). Pyruvate oxidation in 6-week-old fa/fa rats was similar to controls. Remarkably, reduced fat oxidative capacity in 6-week-old fa/fa rats was compensated for by an adaptive increase in intrinsic mitochondrial function at week 12 , which could not be maintained toward week $19\left(140.9 \pm 11.2\right.$ and $57.7 \pm 9.8 \mathrm{nmol} \mathrm{O}_{2} / \mathrm{mg} / \mathrm{min}$, weeks 12 and 19 , respectively), whereas hyperglycemia had developed (13.5 \pm 0.6 and $16.1 \pm 0.3 \mathrm{mmol} / \mathrm{l}$, weeks 12 and 19 , respectively). This mitochondrial adaptation failed to rescue the progressive development of insulin resistance in fa/fa rats. The transition of prediabetes state toward advanced hyperglycemia and hyperinsulinemia was accompanied by a blunted increase in uncoupling protein-3 (UCP3). Thus, in ZDF rats insulin resistance develops progressively in the absence of mitochondrial dysfunction. In fact, improved mitochondrial capacity in hyperinsulinemic hyperglycemic rats does not rescue the progression toward advanced stages of insulin resistance.

Obesity (2010) 18, 1100-1107. doi:10.1038/oby.2009.372

\section{INTRODUCTION}

Skeletal muscle insulin resistance plays a central role in the development of type 2 diabetes (1) and precedes overt type 2 diabetes, often by decades. In studies in patients with overt type 2 diabetes, dysregulation of carbohydrate, and lipid metabolism $(2-5)$ has been reported at whole body level as well as at the level of skeletal muscle. A reduced oxidative capacity in muscle of type 2 diabetic patients, reflected by reduced activity and content of oxidative enzymes (2,6-8), suggests that the observed reduction in oxidative capacity is at the mitochondrial level. Indeed, noninvasive ${ }^{31} \mathrm{P}-\mathrm{MRS}$ revealed prolonged postexercise phosphocreatine resynthesis rates-indicative of reduced mitochondrial oxidative function-in type 2 diabetic patients compared with BMI matched but otherwise healthy controls (9). This decline in mitochondrial oxidative capacity in type 2 diabetic patients correlates with fasting glucose levels and $\mathrm{HbA}_{1 c}$ (9). This may indicate that mitochondrial dysfunction is most pronounced in the more severe type 2 diabetic patients or that hyperglycemia has a negative effect on mitochondrial function, suggesting that hyperglycemia contributes to mitochondrial dysfunction. Furthermore, in insulin-resistant first-degree relatives of type 2 diabetic patients as well as in insulin-resistant elderly, "mitochondrial function" measured as mitochondrial adenosine triphosphate (ATP) synthase flux

${ }^{1}$ NUTRIM School for Nutrition, Toxicology and Metabolism, Maastricht University Medical Centre, Maastricht, The Netherlands; '2Department of Human Movement Sciences, Maastricht University Medical Centre, Maastricht, The Netherlands; ${ }^{3}$ Department of Biomedical Engineering, Biomedical NMR, Eindhoven University of Technology, Eindhoven, The Netherlands; ${ }^{4}$ Department of Human Biology, Maastricht University Medical Centre, Maastricht, The Netherlands. Correspondence: Matthijs K.C. Hesselink (Matthijs.hesselink@bw.unimaas.nl) 
under resting conditions using in vivo ${ }^{31} \mathrm{P}-\mathrm{MRS}$, was declined $(10,11)$, suggesting that reduced mitochondrial function in the "prediabetic state" contributes to the pathogenesis of type 2 diabetes. Recently, using a combined in vivo and ex vivo approach, we extended these findings in first-degree relatives with data indicating declined intrinsic mitochondrial function (12). A recent in vivo study in long-standing type 2 diabetics, however, did not report apparent mitochondrial dysfunction (13), suggesting interaction of the degree of insulin resistance with mitochondrial functional capacity. Thus, we recently examined in vivo mitochondrial function during the progression from the prediabetic state (6-week-old rats) toward a severe hyperglycemic hyperinsulinemic state (12- and 18-week-old rats) in maturing diabetic Zucker diabetic fatty (ZDF) rats (14). In this study, we showed that severe insulin resistance developed in the absence of major signs of mitochondrial dysfunction measured as postexercise phosphocreatine resynthesis rate (14). These findings, however, leave open the option that declined intrinsic mitochondrial function has been compensated for by increased mitochondrial mass. In addition, postexercise phosphocreatine resynthesis rates may be a better reflection of glycolysis derived acetyl-CoA handling than of lipolysis derived acetyl-CoA handling.

To examine whether intrinsic mitochondrial defects are required for the transition of the prediabetic state into severe insulin resistance, we examined mitochondrial function by high-resolution respirometry. Respirometry was performed using a glycolytic (pyruvate), a lipolytic (palmitoyl-CoA and carnitine) and a tricarboxylic acid cycle (glutamate) substrate in mitochondria isolated from skeletal muscle of diabetic ZDF rats $(\mathrm{fa} / \mathrm{fa})$ and their control littermates $(\mathrm{fa} /+)$ at 6,12 , and 19 weeks of age. At 6 weeks, fa/fa rats typically are hyperinsulinemic and normoglycemic and develop severe hyperglycemia (12 weeks of age) with glycosuria at 19 weeks of age, whereas the controls remain normoglycemic and normoinsulinemic during maturation.

\section{METHODS AND PROCEDURES}

\section{Animals}

Twenty-six male, ZDF rats (ZDF/Gmi, fa/fa) and twenty-six heterozygous lean littermates (ZDF/Gmi, fa/+) were purchased from Charles River (Chatillon-sur-Chalaronne, France). Both genotypes arrived at the age of 5 or 11 weeks and were housed in pairs of one genotype, on a 12:12 h light-dark cycle (light from 7:00 AM to 7:00 PM), at room temperature $\left(21-22^{\circ} \mathrm{C}\right)$ with ad libitum access to tap water and diet $(16.7$ energy \% fat, 56.4 energy \% carbohydrates, and 26.8 energy \% protein; Altromin, Lage, Germany).

Body weight and food intake were monitored weekly. Blood samples were obtained weekly in K-EDTA coated tubes via the retro-orbital plexus in $4 \mathrm{~h}$-fasted rats, and centrifuged for $10 \mathrm{~min}$ at $1,000 \mathrm{~g}$. Plasma aliquots were frozen in liquid nitrogen and stored at $-80^{\circ} \mathrm{C}$ until analyses. At 6 , 12 , and 19 weeks of age $(n=8, n=8$, and $n=10$ per age and genotype, respectively), rats were sacrificed for mitochondrial isolation.

All experiments were approved by the Institutional Animal Care and Use Committee.

\section{Blood plasma assays}

Plasma glucose and free-fatty acid values were determined using the hexokinase method (Roche, Basel, Switzerland) and the Wako
NEFA C test kit (Wako Chemicals, Neuss, Germany), respectively. Insulin concentrations were determined using an enzyme-linked immunosorbent assay kit specific for rat insulin (Mercodia, Uppsala, Sweden).

\section{Tissue collection and mitochondrial isolation}

Following $\mathrm{CO}_{2}$ sedation $(<20 \mathrm{~s})$, rats were sacrificed by cervical dislocation. The left tibialis anterior (TA) muscle was rapidly $(<30 \mathrm{~s})$ excised and placed into ice-cold medium containing $100 \mathrm{mmol} / \mathrm{l}$ sucrose, $50 \mathrm{mmol} / \mathrm{l} \mathrm{KCl}, 20 \mathrm{mmol} / \mathrm{l} \mathrm{K}+$ TES, $1 \mathrm{mmol} / \mathrm{l} \mathrm{EDTA}$, and $0.2 \%$ (wt/vol) bovine serum albumin ( $\mathrm{pH}$ 7.4; with $\mathrm{KOH}$ ). The TA was immediately processed for mitochondrial isolation essentially according to Hoeks et al. (15). Meanwhile, the contralateral TA muscle was dissected, trimmed from visible fat and blood, embedded in Tissue-Tek (Sakura Finetek, Zoeterwoude, the Netherlands) and rapidly frozen in liquid nitrogen-cooled isopentane (2-methyl-butane; Fluka, Zwijndrecht, the Netherlands). Samples were stored at $-80^{\circ} \mathrm{C}$ until further analysis.

The protein concentration in the mitochondrial pellet was measured using fluorescamine (Fluram; Fluka, Zwijndrecht, the Netherlands) with bovine serum albumin as a standard (16). Freshly isolated mitochondria were used immediately for mitochondrial respirometry. Remaining mitochondria were stored at $-80^{\circ} \mathrm{C}$ for further analysis.

\section{Mitochondrial respirometry}

Mitochondrial oxygen consumption was measured essentially according to Hoeks et al. (15) at $37^{\circ} \mathrm{C}$ using a two-chamber oxygraph (OROBOROS Instruments, Innsbruck, Austria) and expressed as nmol $\mathrm{O}_{2} / \mathrm{mg}$ mitochondrial protein per minute.

Freshly isolated skeletal muscle mitochondria $(0.2 \mathrm{mg}$ of mitochondrial protein for pyruvate/glutamate and $0.5 \mathrm{mg}$ for carnitine + palmitoyl$\mathrm{CoA}$ ) were incubated in a medium consisting of $100 \mathrm{mmol} / \mathrm{l}$ sucrose, $20 \mathrm{mmol} / \mathrm{l} \mathrm{K}+\mathrm{Tes}(\mathrm{pH} 7.2), 50 \mathrm{mmol} / \mathrm{l} \mathrm{KCl}, 2 \mathrm{mmol} / 1 \mathrm{MgCl}_{2}, 1 \mathrm{mmol} / \mathrm{l}$ EDTA, $4 \mathrm{mmol} / 1 \mathrm{KH}_{2} \mathrm{PO}_{4}, 3 \mathrm{mmol} / \mathrm{l}$ malate, and $0.1 \%$ of bovine serum albumin.

Substrates used to feed mitochondrial respiration were: $5 \mathrm{mmol} / \mathrm{l}$ pyruvate (pyr), $10 \mathrm{mmol} / \mathrm{l}$ glutamate (glut) (glycolytic substrates), and $2 \mathrm{mmol} / \mathrm{l}$ carnitine $+50 \mu \mathrm{mol} / \mathrm{l}$ palmitoyl-CoA (fatty acid substrate). This allows us to examine the functionality of the pyruvate carrier system, pyruvate dehydrogenase complex (pyr), the glutamate dehydrogenase (glut), as well as the functionality of the carnitine acyl transferase complex and subsequent $\beta$-oxidation.

After measuring adenosine diphosphate (ADP) driven state 3 respiration by addition of $450 \mu \mathrm{mol} / \mathrm{ADP}$, oligomycin was added to the chamber to block the mitochondrial $\mathrm{F}_{1}-\mathrm{F}_{0}$ ATPase to examine the residual respiration reflecting proton leak (state $4 \mathrm{O}$ ). Titration with $0.5 \mu \mathrm{mol} / \mathrm{l}$ additions of the chemical uncoupler carbonyl cyanide p-trifluoromethoxyphenylhydrazone (FCCP) was used to measure maximal mitochondrial respiratory capacity (state uncoupled, state u). All substrates were dissolved in double-distilled water whereas FCCP and oligomycin were dissolved in $96 \%$ ethanol. Pilot experiments showed that ethanol itself did not have any effects on the parameters measured. Traces were randomized. Addition of excess cytochrome $\mathrm{C}$ to the chamber in the presence of pyruvate and ADP did not increase respiration (data not shown); indicating that during isolation outer mitochondrial membrane integrity was maintained. The respiratory control ratio was calculated as state 3/state 4O. A representative trace of oxygen consumption of isolated mitochondria from anterior tibialis muscle of 12 -week-old lean rats respiring on pyruvate is shown in the Supplementary Figure S1 online.

\section{Western blotting analysis of mitochondrial and oxidized proteins}

To test markers of mitochondrial density of the muscles processed for mitochondrial isolation, western blot analyses were performed in muscle homogenates of the contralateral TA muscle using an antibody cocktail (MS601; MitoSciences, Eugene, OR) against structural components of each of the five oxidative phosphorylation complexes 
(i.e., to the ND6 subunit of complex I, the $30 \mathrm{kDa}$ Ip subunit of complex II, the $47 \mathrm{kDa}$ core protein 2 of complex III, subunit II of cytochrome $\mathrm{C}$ oxidase (COXII, complex IV) and the a-subunit of the $\mathrm{F}_{1} \mathrm{~F}_{0}$ ATP synthase (complex V)).

In the whole muscle homogenates, we also analyzed uncoupling protein-3 (UCP3) protein content by western blotting essentially according to Hoeks et al. (17).

To determine superoxide-derived changes in protein carbonylation, we performed an assay for carbonylated proteins provided by Chemicon (Oxyblot oxidized protein detection kit; Millipore, Amsterdam, the Netherlands,). Derivatization of 2,4-dinitrophenylhydrazine was carried out for $15 \mathrm{~min}$ following manufacturer's instruction. In brief, the carbonyl groups in the protein side chains are derivatized to 2,4-dinitrophenylhydrazone (DNP-hydrazone) by reaction with 2,4-dinitrophenylhydrazine. The DNP-derivatized protein samples are subjected to gel electrophoresis and subsequent western blotting. Densitometry of the 2,4-dinitrophenylhydrazine-derived bands in the gel was performed and normalized to a standard control sample loaded on all gels and expressed as arbitrary units (AU).

In isolated mitochondrial fractions western blotting against the adenine nucleotide translocator 1 (ANT1) was performed. Upon homogenization in lysis buffer, containing 1\% NP40, $0.5 \%$ sodium dodecyl sulfate, $1 \mathrm{mmol} / \mathrm{l}$ phenylmethylsulfonyl fluoride, and $4 \% \mathrm{com}$ plete inhibitor stock, lysates were processed for western blotting using a monoclonal antibody against ANT1 (MSA02; MitoSciences). After incubation with the appropriate secondary antibodies, protein specific bands were detected and quantified with Odyssey Infrared Imager (LICOR; Westburg, Leusden, the Netherlands) and expressed as AU.

\section{Muscle fiber typing}

Mitochondrial function examined in vivo, also relies on the presence of substrates and extramitochondrial enzyme and transport capacity of the muscle. These characteristics are known to vary widely across different muscle fiber types (oxidative type I fibers vs. more glycolytic type IIa and IIb fibers), even within the same muscle. Our mitochondrial isolation procedure results in a mixture of mitochondria originating from all fiber types. Thus, we monitored muscle fiber typology throughout the study by immunofluorescence in contralateral TA muscle samples using monoclonal antibodies against the fiber type specific myosin heavy chain isoforms (A4.840 against myosin heavy chain I and N2.261 against myosin heavy chain IIa, developed by Dr H.M. Blau, obtained from the Developmental Studies Hybridoma Bank, Iowa City, IA). Myosin heavy chain IIb fibers remained unstained. Staining and subsequent analyses was performed essentially according to ref. 18 .

\section{Statistics}

All results are reported as mean \pm s.e.m. Statistical analyses were performed two-sided using SPSS for Windows 15.0 software (SPSS, Chicago, IL) and statistical significance was set at $P<0.05$. For comparison of respiratory data between genotypes at 6 weeks of age unpaired $t$-tests were performed. To examine the age- $(6,12,19$ weeks of age) and genotypeeffects (fa/fa vs. control) as well as interaction (age $\times$ genotype) effects on the respiratory data of the three age groups, a two-way ANOVA $(2 \times$ 3 factorial experiment), univariate model was performed. In case of significance of the age $\times$ genotype interaction parameter, Bonferroni corrected post hoc tests were carried out for split genotype groups.

\section{RESULTS}

\section{Animal characteristics}

Body mass and food intake were significantly higher in the $\mathrm{fa} / \mathrm{fa}$ than in the control rats $(P<0.001$ at all ages $)$. At 6 weeks of age body mass equaled $193.7 \pm 1.9 \mathrm{~g}$ vs. $147.2 \pm 3.3 \mathrm{~g}$ ( $\mathrm{fa} / \mathrm{fa}$ vs. control), at 12 weeks $345.0 \pm 5.3 \mathrm{~g}$ vs. $284.0 \pm 10.1 \mathrm{~g}$ and at the age of 19 weeks $372.5 \pm 5 \mathrm{~g}$ vs. $335.4 \pm 6.2 \mathrm{~g}$, which is well in accord with recognized values reported in literature for the ZDF model $(19,20)$.

At the onset of the study, blood glucose levels were slightly but significantly higher in fa/fa rats as compared to control rats at week $6(4.6 \pm 0.1$ vs. $3.9 \pm 0.1 \mathrm{mmol} / \mathrm{l})$ and rapidly increased in the $\mathrm{fa} / \mathrm{fa}$ rats in week $12(13.5 \pm 0.6 \mathrm{mmol} / \mathrm{l})$ and week $19(16.1 \pm 0.3 \mathrm{mmol} / \mathrm{l})$, while remaining normal in the control rats $(3.8 \pm 0.2 \mathrm{mmol} / \mathrm{l}$ and $4.0 \pm 0.3 \mathrm{mmol} / \mathrm{l}$ at week 12 and 19, respectively). From week 6 onward insulin levels in $\mathrm{fa} / \mathrm{fa}$ rats were significantly higher than in control rats $(1.6 \pm$ $0.4 \mu \mathrm{g} / \mathrm{l}$ vs. $0.2 \pm 0.0 \mu \mathrm{g} / \mathrm{l}, 1.6 \pm 0.3 \mu \mathrm{g} / \mathrm{l}$ vs. $0.4 \pm 0.1$, and $0.8 \pm$ $0.1 \mu \mathrm{g} / \mathrm{l}$ vs. $0.3 \pm 0.1 \mu \mathrm{g} / \mathrm{l}$ at 6,12 , and 19 weeks, respectively). Thus, at week 6 the fa/fa rats were hyperinsulinemic and normoglycemic, reflecting a prediabetic insulin-resistant state. At the age of 12 weeks, fa/fa rats showed severe hyperglycemia with hyperinsulinemia, indicative of severe insulin resistance, whereas at 19 weeks of age fa/fa rats still suffered from severe hyperglycemia while insulin levels started to decline, indicative of type 2 diabetes with imminent pancreas failure. No such changes were observed in the normoglycemic, normoinsulinemic control rats.

Free-fatty acid levels were comparable throughout the study $(181.0 \pm 19.8 \mu \mathrm{mol} / \mathrm{l}$ vs. $165.6 \pm 19.9 \mu \mathrm{mol} / \mathrm{l}, 180.4 \pm 18.9 \mu \mathrm{mol} / \mathrm{l}$ vs. $206.2 \pm 24.9 \mu \mathrm{mol} / \mathrm{l}$, and $172.3 \pm 14.5 \mu \mathrm{mol} / \mathrm{l}$ vs. $192.8 \pm$ $33.4 \mu \mathrm{mol} / \mathrm{l}$, at 6,12 , and 19 weeks for fa/fa vs. the controls, respectively).

\section{Mitochondrial respirometry}

Respiratory control ratios on pyruvate yielded high-respiratory control ratio values (respiratory control ratio $14.0 \pm 1.7$ and $14.0 \pm 1.8$ in 6-week-old fa/fa vs. lean rats, $12.9 \pm 2.3$ and $12.2 \pm 1.8$ in 12-week-old fa/fa vs. lean rats and $12.4 \pm 2.3$ and $11.7 \pm 2.1$ in 19 -week-old fa/fa vs. lean rats), indicative of high quality mitochondria (21).

Genotype differences for state 3, state 40 , and state u respiration at week 6 were examined for baseline differences. On pyruvate, no significant differences were found between $\mathrm{fa} / \mathrm{fa}$ and control rats for any of these states $(P=0.61, P=0.39, P=0.56$ for state 3, 4, and u, respectively) (Figure 1a,b). Similar results were observed on glutamate (data not shown). Respiration on palmitoyl-CoA in presence of carnitine, however, was significantly lower in hyperinsulinemic fa/fa rats than in normoinsulinemic control rats at 6 weeks of age. Both ADP-driven state $3(62.3 \pm 9.5$ vs. $119.1 \pm 13.8 \mathrm{nmol} \mathrm{O} / \mathrm{mg}$ mitochondrial protein/min, $P=0.008$ ) and FCCP-stimulated maximal respiration state $\mathrm{u}$ respiration $(87.7 \pm 13.3$ vs. $141.9 \pm 14.3 \mathrm{nmol}$ $\mathrm{O}_{2} / \mathrm{mg}$ mitochondrial protein/min, $P=0.018$ ) were $\sim 50 \%$ lower in fa/fa rats compared with control rats (Figure 1c,d). No differences in state $4 \mathrm{O}$ respiration between genotypes were observed at 6 weeks of age $(P=0.47)$.

Subsequently, we examined mitochondrial respiration in time. ADP-driven state 3 respiration on pyruvate significantly changed with age $(P=0.002)$ with a tendency of an age $\times$ genotype interaction $(P=0.129)$ (Figure 1a). Bonferroni post hoc testing revealed a tendency to increased state 3 respiration from 6 to 12 weeks of age $(P=0.061)$ followed by a profound and significant 

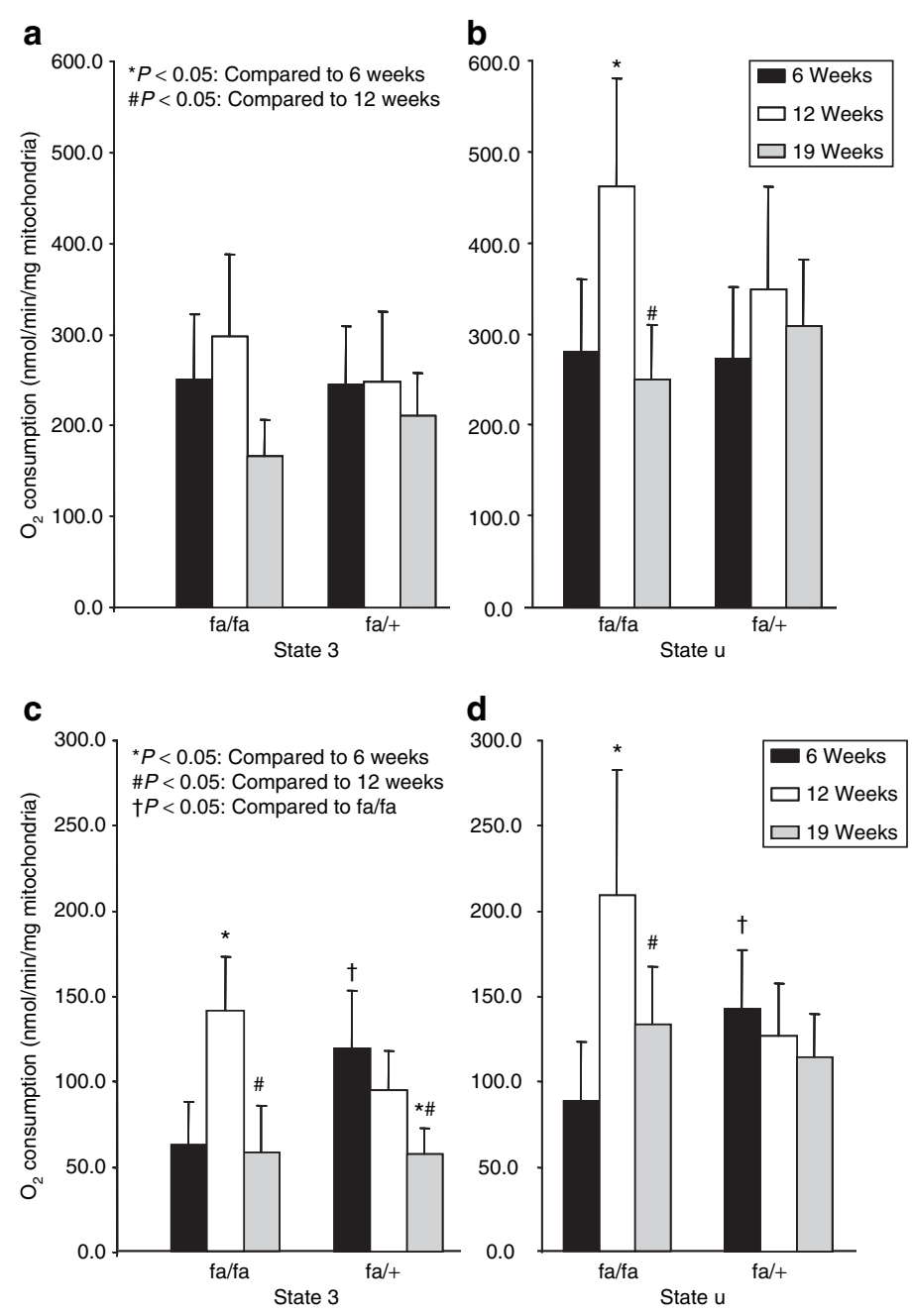

Figure 1 Oxidative capacity of isolated mitochondria from TA muscle respiring on (a) state 3 (maximal ADP-driven respiration limited by the capacity of the oxidative phosphorylation system) on pyruvate and (b) state $u$ (maximal ADP-driven respiration not limited by the oxidative phosphorylation system) pyruvate; (c) state 3 on palmitoyl-CoA and (d) state $\mathrm{u}$ on palmitoyl-CoA in fa/fa (black bars) and control (white bars) rats at 6,12 , and 19 weeks. Error bars represent s.e.m. Significance and age/genotype represented as legend in the figure. ADP, adenosine diphosphate; TA, tibialis anterior.

decline from 12 to 19 weeks of age $(P=0.002)$ in the fa/fa rats, whereas these changes were insignificant in nondiabetic control rats $(P=0.384)$. State $4 \mathrm{O}$ respiration on pyruvate significantly changed with age $(P=0.011)$, with a significant age $\times$ genotype effect $(P=0.043)$. State $4 \mathrm{O}$ respiration decreased significantly from 12 to 19 weeks in the fa/fa rats $(P=0.003)$, whereas no change with age was observed in control rats $(P=0.880)$. State $\mathrm{u}$ also significantly changed with age $(P<0.001)$, with a significant age $\times$ genotype effect $(P=0.026)$ (Figure $1 b)$. Like state 3 , state u increased significantly from 6 weeks to 12 weeks $(P=0.001)$ of age in fa/fa rats, with a subsequent decrease from 12 to 19 weeks $(P=0.000)$. For the control rats no significant change during maturation was observed $(P=0.266)$. Respiration on glutamate qualitatively resulted in the same profiles as observed for pyruvate respiration (data not shown).

For palmitoyl-CoA-stimulated respiration, in the presence of carnitine, state 3 respiration significantly changed with age $(P<0.001)$, with a significant age $\times$ genotype effect $(P<0.001)$
(Figure 1c). State 3 respiration increased significantly from week 6 to $12(P<0.001)$, with a subsequent significant decline toward week $19(P<0.001)$ in late stage diabetic fa/fa rats. For control rats, however, a steady significant age-related decline from week 6 to $19(P<0.001)$ was observed for state 3 respiration. State $4 \mathrm{O}$ respiration on palmitoyl-CoA was unaffected by age or genotype. State u respiration mimicked the profile observed for state 3 respiration in the fa/fa rats; significant changes with age $(P=0.003)$ and a significant age $\times$ genotype effect $(P<0.001)$ (Figure 1d). As for state 3 , in fa/fa rats state u respiration significantly increased from 6 to 12 weeks of age $(P<0.001)$ with a subsequent decline toward 19 weeks of age $(P=0.013)$. In control rats state $\mathrm{u}$ was not significantly different across age groups.

\section{Mitochondrial density markers}

Structural components of oxidative phosphorylation complexes in whole muscle homogenates were comparable between $\mathrm{fa} / \mathrm{fa}$ 


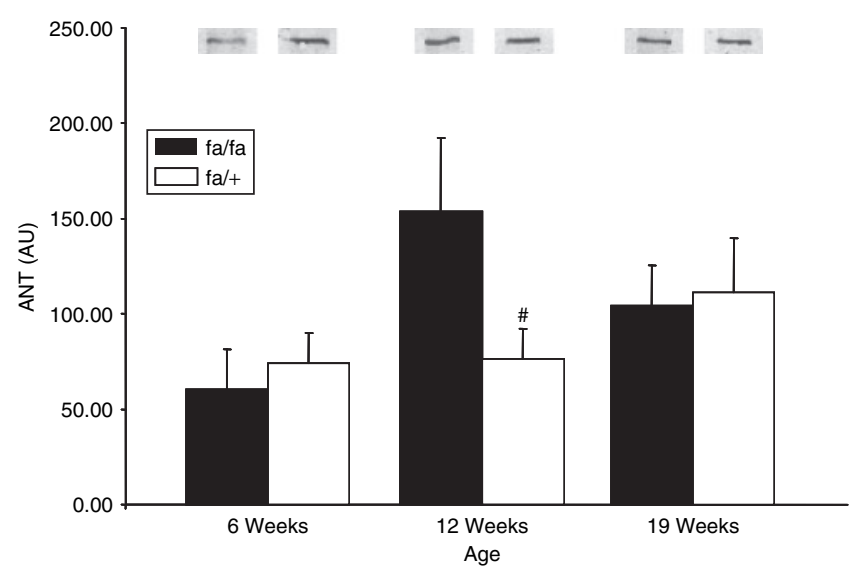

Figure 2 Arbitrary units of protein expression levels of ANT, measured in isolated TA mitochondrial fractions, in fa/fa (black bars) and control (white bars) rats at 6,12 , and 19 weeks of age. Error bars represent s.e.m. "Significant difference from control rats using nonparametric Mann-Whitney U test. ANT, adenine nucleotide transporter; AU, arbitrary units; TA, tibialis anterior.

rats and control rats and not different between age groups for any of the components examined. Joint examination of total protein expression of complex I-V also did not hint toward differences in mitochondrial density $(122 \pm 21$ vs. $124 \pm 15$, $93 \pm 20$ vs. $81 \pm 12$ and $92 \pm 16$ vs. $97 \pm 14 \mathrm{AU}$ for fa/fa vs. control rats at 6,12 , and 19 weeks of age, respectively).

\section{ANT1, UCP3, and carbonylated proteins}

Protein levels of the mitochondrial ADP-ATP translocator ANT1 were measured in mitochondria remaining after respirometry. As a result ANT1 protein data were successfully analyzed in most but not all mitochondrial suspensions; in 6-week-old rats data shown are based on $n=6$ for fa/fa rats and $n=4$ for control rats. We hence were unable to run the two-way ANOVA univariate model on these data, rather we used nonparametric Mann-Whitney $U$ tests for genotype comparisons on 6,12, and 19-week-old fa/fa and control rats. In 12-weekold rats ANT1 protein expression was significantly higher in $\mathrm{fa} / \mathrm{fa}$ rats compared to control rats ( $154 \pm 38$ vs. $76 \pm 16 \mathrm{AU}$, respectively, $P<0.05)$, whereas at 19 weeks ANT1 values were comparable (105 \pm 21 vs. $111 \pm 29 \mathrm{AU}$, respectively, $P=0.418)$ (Figure 2).

UCP3 protein expression significantly increased with age $(P<0.001)$ with a significant genotype effect $(P=0.007)$ and a significant age $\times$ genotype interaction $(P<0.001)$. Bonferroni post hoc analyses revealed that the increase in UCP3 was blunted in diabetic fa/fa rats, with significantly lower levels at 19 weeks of age for fa/fa rats compared to control rats (54 \pm 4 vs. $51 \pm 7,78 \pm 8$ vs. $80 \pm 7$, and $111 \pm 7$ vs. $171 \pm 11 \mathrm{AU}$ for $\mathrm{fa} / \mathrm{fa}$ vs. control rats at 6,12 , and 19 weeks of age, respectively) (Figure 3).

The presence of carbonylated proteins as identified by the Oxyblot procedure was similar in both genotypes and no differences were detected with age ( $1.31 \pm 0.7$ vs. $1.01 \pm 0.4,1.28 \pm$ 0.5 vs. $1.00 \pm 0.5,1.32 \pm 0.5$ vs. $1.29 \pm 0.5 \mathrm{in} \mathrm{fa/fa} \mathrm{vs.} \mathrm{control} \mathrm{rats}$ at 6,12 , and 19 weeks of ages, respectively).

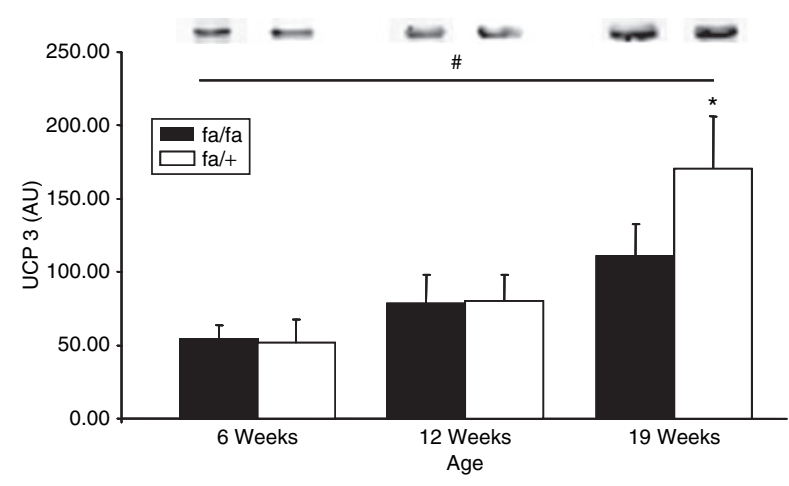

Figure 3 Arbitrary units of protein expression levels of UCP3, measured in whole TA muscle homogenates, in fa/fa (black bars) and control (white bars) rats at 6,12 , and 19 weeks of age. "Significant age-effect, *control rats significant higher compared with fa/fa rats $(P<0.05)$. Error bars represent s.e.m. TA, tibialis anterior; UCP3, uncoupling protein-3.

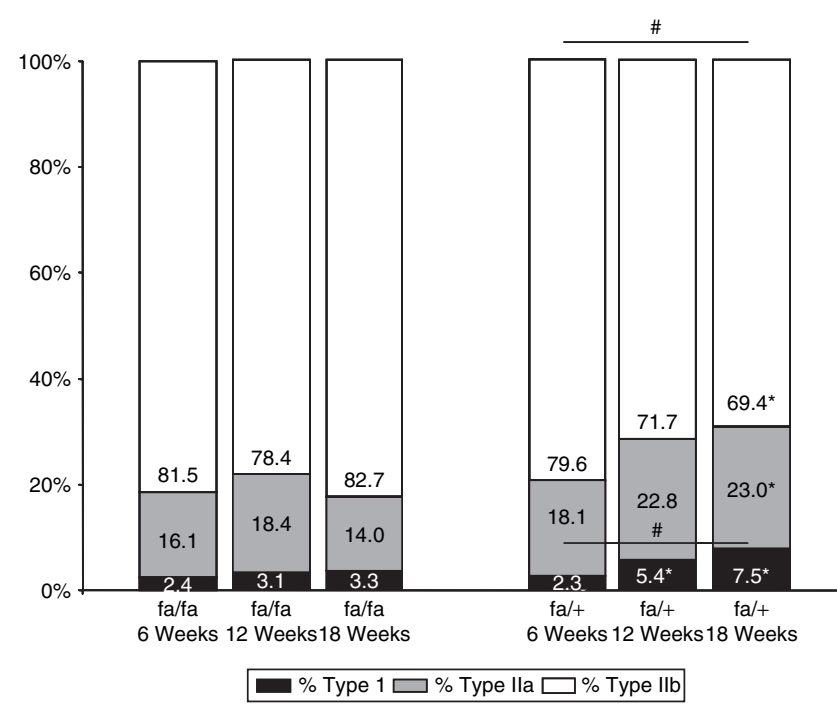

Figure 4 Fiber type composition (\%) (type I, type lla, and typell b) of the TA muscle at 6,12 , and 19 weeks of age. "Significant age-effect for fiber type I and type Ilb in the control rats $(P<0.05)$. The age-related increase in oxidative fibers (type I) at the expense of glycolytic fibers (type Ila and IIb) is blunted during progression of insulin resistance in the fa/fa rats. ${ }^{*}$ Control rats significant higher compared with fa/fa rats $(P<0.05)$. Significance and fiber type represented as legend in the figure. TA, tibialis anterior.

\section{Muscle fiber typing}

Analyses of on average $947 \pm 61$ muscle fibers per rat in the TA muscle showed a progressive increase in the percentage type I fibers at the expense of type IIb fibers in control rats. In fa/fa rats these changes were absent (Figure 4), resulting in a significantly lower percentage type I fibers and a higher percentage of type IIb fibers in fa/fa rats compared to control rats at 12 and 19 weeks of age.

\section{DISCUSSION}

In a recognized model of progressive insulin resistance and development of type 2 diabetes during maturation-the ZDF rat (20) - we examined whether transition from the prediabetic 
state toward severe insulin resistance requires intrinsic defects in muscle mitochondrial function.

In mitochondria isolated from TA muscle of ZDF rats (fa/fa), as well as in normoglycemic normoinsulinemic control rats, mitochondrial function was assessed at the age of 6, 12, and 19 weeks. At none of the ages we observed significant differences in marker proteins for mitochondrial content. Interestingly, palmitoyl-CoA driven mitochondrial respiration was lower in 6-week-old fa/fa rats than in control rats, whereas at that age mitochondrial respiration on pyruvate and glutamate was similar in both genotypes, suggesting a specific defect in fat oxidative capacity. Remarkably, at 12 weeks of age, fa/fa rats showed an adaptive increase in state 3 respiration on palmitoyl-CoA and in state u respiration on both palmitoyl-CoA and pyruvate. This increase in mitochondrial capacity was not maintained, indicated by a drop in respiratory capacity at 19 weeks of age, resulting in the same level of respiration in 19-week-old diabetic fa/fa rats as in lean control rats. Taken together, in fa/fa rats lower palmitoyl-CoA respiration was observed in young hyperinsulinemic normoglycemic rats, followed by an (adaptive) improvement in mitochondrial capacity during the development of type 2 diabetes, which returned to levels similar to control animals when type 2 diabetes progressed to an advanced state.

Most (9-11,22,23), but not all (13), in vivo studies using nuclear magnetic resonance spectroscopy revealed "mitochondrial dysfunction" in type 2 diabetic patients as well as in prediabetic subjects. The observation in the prediabetic state suggests that aberrations in mitochondrial capacity (either due to the decreased mitochondrial content or compromised intrinsic mitochondrial function) may underlie the pathogenesis of type 2 diabetes. Here we show that in fa/fa rats, during the transition of the prediabetic state toward full-blown type 2 diabetes, marker proteins of mitochondrial density were unaltered and comparable to normoglycemic and normoinsulinemic control rats. This is in line with data in human prediabetic and type 2 diabetic subjects compared to age and BMI-matched normoglycemic controls, who also show no differences in the muscular protein content of complex I-V or cytochrome C (24). In these studies mitochondrial mass was examined by marker proteins of mitochondrial density or classical enzymatic assays supposed to reflect mitochondrial mass. Part of these markers for mitochondrial mass may as well be a reflection of intrinsic mitochondrial function.

To examine the role of intrinsic mitochondrial function in insulin resistance related mitochondrial dysfunction, we measured intrinsic mitochondrial respiratory capacity in isolated mitochondria. We show that in 6-week-old fa/fa rats ADPdriven state 3 and FCCP-stimulated maximal palmitoyl-CoA respiration was reduced compared to control rats. At this age, $\mathrm{fa} / \mathrm{fa}$ rats are hyperinsulinemic but normoglycemic and had a higher body weight compared to their lean controls. Although we did not perform glucose or insulin tolerance tests or hyperinsulinemic euglycemic clamps in these rats, it should be noted that the ZDF rat is a well-established model of progressive insulin resistance (20). Given the severe hyperglycemia and hyperinsulinemia reported in here at the age of 12 weeks, we have good reason to expect that the current set of animals indeed are insulin resistant. Interestingly, already at the age of 12 weeks intramyocellular lipid (IMCL) levels are markedly elevated in fa/fa rats compared to control rats (14). These data match data obtained in prediabetic patients, in which elevated IMCL levels are accompanied by a lower mitochondrial function $(11,12)$. Importantly, however, the lower state 3 and state $\mathrm{u}$ respiration was observed only on palmitoyl-CoA while respiration was similar between genotypes using glutamate or pyruvate as glycolytic substrates. This suggests that a specific defect in fat oxidative capacity ( $\beta$-oxidation) or mitochondrial import of fatty acylCoA esters via the carnitine palmitoyl transferase system may be associated with insulin resistance in $\mathrm{fa} / \mathrm{fa}$ rats. Recently, a link has been made between incomplete $\beta$-oxidation of long-chain fatty acids, resulting in increased levels of acylcarnitines and insulin resistance (25). In this respect it is of importance to note that in our model of respirometry in isolated mitochondria palmitoyl-CoA and carnitine are provided in excess and that respiration in state $\mathrm{u}$ is not limited by the oxidative phosphorylation system, and hence reflects the maximal capacity of the electron transport chain to reduce palmitoyl-CoA-derived redox equivalents. This model hence does not permit conclusions regarding complete or incomplete $\beta$-oxidation of palmitoyl-CoA. It does, however, fit with the hypothesis of Koves et al. as indicated by the lower capacity to oxidize palmitoyl-CoA in prediabetic 6-week-old fa/fa rats, which probes mitochondria for incomplete $\beta$-oxidation compared to control rats. To examine whether this reduction in fat oxidative capacity (partly) underlies the accumulation of IMCL and indeed contributes to the development of insulin resistance, requires measurements at an earlier age than performed in the present study.

As fa/fa rats lack the leptin receptor and leptin stimulates skeletal muscle fat oxidation $(26,27)$, the reduced fat oxidative capacity at early age in fa/fa rats might be due to the ablation of the leptin receptor. This makes this model suitable to investigate the consequence of reduced fat oxidation on the development of type 2 diabetes. In that context, we observed that the initial reduction in fat oxidative capacity was followed by an unanticipated compensatory improvement in mitochondrial respiration on a lipolytic substrate in fa/fa rats compared to controls. At 12 weeks of age, hyperinsulinemia and hyperglycemia in fa/fa rats coincided with significant increases in state $\mathrm{u}$ respiration both on pyruvate and on palmitoyl-CoA. In state 3 this increase only reached significance on palmitoyl-CoA. We interpret this improved intrinsic aspect of mitochondrial function on week 12 as an adaptive response of the fa/fa rats to the sustained increased availability of substrates due to hyperphagia, hyperglycemia and/or elevated IMCL levels. In line with this, it has been shown that rats fed a high-fat diet improve their mitochondrial capacity to deal with the elevated supply of fat to the muscle (28).

We consistently observed the FCCP uncoupled state u respiration to exceed state 3 respiration, implicating that the capacity of the phosphorylation system (mainly determined by $F_{1}-F_{0}$ ATPase and ANT1 activity) controls maximal flow 
through the electron transport chain. Because improvements in mitochondrial respiration were observed in state $u$ (when the phosphorylation system is bypassed due to the use of FCCP), adaptations at the level proximal to the phosphorylation system are the most likely explanation for this observation. As similar effects are observed for state $u$ on pyruvate and palmitoyl-CoA, both relying on distinct substrate transporting systems, these observations indicate that improvements in electron transport chain capacity primarily underlie the improved respiration at week $12 \mathrm{in} \mathrm{fa/fa} \mathrm{rats.} \mathrm{Interestingly,}$ our in vivo study in the same rat model showed prolonged phosphocreatine resynthesis rates (declined mitochondrial function) at week $12 \mathrm{in} \mathrm{fa/fa} \mathrm{rats} \mathrm{(14),} \mathrm{suggesting} \mathrm{that} \mathrm{in} \mathrm{vivo}$ mitochondrial function in 12 -week-old $\mathrm{fa} / \mathrm{fa}$ rats may be depressed due to defects upstream the pyruvate dehydrogenase reaction. These defects are bypassed in the ex vivo respirometry assay presented here. In addition, we also observed a significant increase in protein content of ANT1 in mitochondria isolated the fa/fa rats from 6 to 12 weeks of age. The increase in ANT1 may serve to facilitate the increased capacity of the electron transport chain systems, allowing increased state 3 respiration.

At week 19 of age, when type 2 diabetes develops to an advanced state, the adaptive mitochondrial response observed in fa/fa rats, however, has disappeared entirely and respiratory capacity and ANT1 levels drop to values similar to those observed in control rats. From the present study, it cannot be deduced if the decline in mitochondrial function between weeks 12 and 19 contributes to the augmentation of insulin resistance, or rather is a consequence of a further aggravation of the diabetic state. Our data seem to indicate, however, that the mitochondrial system is capable of adaptive responses and that diabetes-induced changes in mitochondrial respiration are more likely determined by secondary effects of sustained hyperglycemia or elevated IMCL levels. The reason for the inability to maintain high levels of mitochondrial respiration in the face of high levels of IMCL should be topic of further study.

Previously, we observed declined levels of the mitochondrial UCP3 in type 2 diabetes patients (29) and hypothesized that UCP3 is involved in the maintenance of mitochondrial function under diabetogenic conditions by lowering superoxide production via mild uncoupling (30). In the current model, we interestingly observed a gradual increase of UCP3 during maturation in control rats. This increase, however, was significantly blunted in the model of progressive insulin resistance in fa/fa rats, resulting in significantly lower levels of UCP3 at week 19 in diabetic rats compared to control rats. This is consistent with our previous observation that UCP3 protein content is reduced by $50 \%$ in type 2 diabetic patients compared to BMI-matched controls (24). Despite this consistency, both the origin as the consequence of the blunted increase in UCP3 in $\mathrm{fa} / \mathrm{fa}$ rats remains to be established and the differences in UCP3 protein expression were not reflected in differences in the expression of carbonylated proteins, an indirect marker of superoxide production. By measuring live production of superoxide using electron spin resonance in freshly isolated mitochondria we recently proved that the age-related increase in mitochondrial superoxide production was blunted in mice overexpressing UCP3 (31) This indicates that increasing UCP3 indeed may lower superoxide production. Why this potential of UCP3 to lower superoxide production apparently is not reflected in carbonylated proteins in the current model of a blunted increase in diabetic rats remains to be established.

Changes in muscle fiber type may also contribute to the changes in whole body oxidative capacity. We observed a shift in muscle fiber typology in the control rats with age, resulting in a more oxidative muscle fiber with more type I and less type IIb fibers. This age-related switch was completely absent in diabetic rats during the progression of the disease. This implies that also the extramitochondrial handling of oxidative substrates like fatty acids becomes compromised, which may contribute to the accumulation of insulin signaling desensitizing lipid intermediates (32).

The present study examined skeletal muscle intrinsic mitochondrial function in relation to a complex disease state originating from defects in multiple organs. Although skeletal muscle insulin sensitivity is a major determinant of whole body-insulin resistance, the exact implication of our findings to the in vivo situation requires further examination.

In conclusion, the present study shows that fat oxidative capacity is reduced in young, insulin resistant fa/fa rats, which is compensated for by improved mitochondrial capacity at 12 weeks of age, even though fa/fa rats develop hyperglycemia in this period. This suggests that, in this rat model, intrinsic muscular mitochondrial dysfunction is not required for the transition of the prediabetic state into full-blown type 2 diabetes. We do show, however, that during maturation and in the presence of hyperinsulinemia and hyperglycemia the initial adaptive response in mitochondrial function is not maintained toward week 19 of age in fa/fa rats, when advanced type 2 diabetes develops. The blunted increase of UCP3 and the shift toward a more glycolytic muscle fiber typology between week 12 and 19 are likely to contribute to aggravation of the diabetic state in the diabetic $\mathrm{fa} / \mathrm{fa}$ rats.

\section{SUPPLEMENTARY MATERIAL}

Supplementary material is linked to the online version of the paper at http://www.nature.com/oby

\section{ACKNOWLEDGMENTS}

We thank Esther Moonen-Kornips, Denis van Beurden, and Jordy Gatignon for excellent technical assistance. The work of Dr Hesselink is supported by a VIDI grant for innovative research (917.66.359). E. Lenaers is supported by a grant from the School of Life Sciences.

\section{(C) 2009 The Obesity Society}

\section{REFERENCES}

1. Petersen KF, Shulman Gl. Etiology of insulin resistance. Am J Med 2006;119:S10-S16.

2. Simoneau JA, Kelley DE. Altered glycolytic and oxidative capacities of skeletal muscle contribute to insulin resistance in NIDDM. J Appl Physiol 1997;83:166-171

3. Kelley DE, Mintun MA, Watkins SC et al. The effect of non-insulin-dependent diabetes mellitus and obesity on glucose transport and phosphorylation in skeletal muscle. J Clin Invest 1996;97:2705-2713. 
4. Kelley D, Mokan M, Veneman T. Impaired postprandial glucose utilization in non-insulin-dependent diabetes mellitus. Metab Clin Exp 1994;43: 1549-1557.

5. Kelley DE, Simoneau JA. Impaired free fatty acid utilization by skeletal muscle in non-insulin-dependent diabetes mellitus. J Clin Invest 1994;94:2349-2356.

6. Simoneau JA, Veerkamp JH, Turcotte LP, Kelley DE. Markers of capacity to utilize fatty acids in human skeletal muscle: relation to insulin resistance and obesity and effects of weight loss. Faseb J 1999;13:2051-2060.

7. Kruszynska YT, Mukherjee R, Jow L et al. Skeletal muscle peroxisome proliferator- activated receptor-gamma expression in obesity and noninsulin-dependent diabetes mellitus. J Clin Invest 1998;101:543-548.

8. Kruszynska YT, Mulford MI, Baloga J, Yu JG, Olefsky JM. Regulation of skeletal muscle hexokinase II by insulin in nondiabetic and NIDDM subjects. Diabetes 1998;47:1107-1113.

9. Schrauwen-Hinderling VB, Kooi ME, Hesselink MK et al. Impaired in vivo mitochondrial function but similar intramyocellular lipid content in patients with type 2 diabetes mellitus and BMI-matched control subjects. Diabetologia 2007;50:113-120.

10. Petersen KF, Befroy D, Dufour S et al. Mitochondrial dysfunction in the elderly: possible role in insulin resistance. Science 2003;300:1140-1142.

11. Petersen KF, Dufour S, Befroy D, Garcia R, Shulman Gl. Impaired mitochondrial activity in the insulin-resistant offspring of patients with type 2 diabetes. N Engl J Med 2004;350:664-671.

12. Phielix E, Schrauwen-Hinderling VB, Mensink $M$ et al. Lower intrinsic ADP-stimulated mitochondrial respiration underlies in vivo mitochondrial dysfunction in muscle of male type 2 diabetic patients. Diabetes 2008;57:2943-2949.

13. De Feyter HM, van den Broek NM, Praet SF et al. Early or advanced stage type 2 diabetes is not accompanied by in vivo skeletal muscle mitochondrial dysfunction. Eur J Endocrinol 2008;158:643-653.

14. De Feyter HM, Lenaers E, Houten SM et al. Increased intramyocellular lipid content but normal skeletal muscle mitochondrial oxidative capacity throughout the pathogenesis of type 2 diabetes. FASEB J 2008;22: 3947-3955

15. Hoeks J, Briedé JJ, de Vogel J et al. Mitochondrial function, content and ROS production in rat skeletal muscle: effect of high-fat feeding. FEBS Lett 2008;582:510-516

16. Udenfriend S, Stein S, Böhlen $P$ et al. Fluorescamine: a reagent for assay of amino acids, peptides, proteins, and primary amines in the picomole range. Science 1972;178:871-872.

17. Hoeks J, Hesselink MK, van Bilsen M et al. Differential response of UCP3 to medium versus long chain triacylglycerols; manifestation of a functional adaptation. FEBS Lett 2003;555:631-637.
18. Minnaard R, Drost MR, Wagenmakers AJ et al. Skeletal Muscle wasting and contractile performance in septic rats. Muscle Nerve 2005;31:339-348.

19. Peterson RG, Shaw WN, Neel M, Little LA, Eichberg J. Zucker diabetic fatty rat as a model for non-insulin-dependent diabetes mellitus. ILAR News 1990;32:16-19.

20. Etgen GJ, Oldham BA. Profiling of Zucker diabetic fatty rats in their progression to the overt diabetic state. Metab Clin Exp 2000;49:684-688.

21. Rasmussen HN, Rasmussen UF. Small scale preparation of skeletal muscle mitochondria, criteria of integrity, and assays with reference to tissue function. Mol Cell Biochem 1997;174:55-60.

22. Morino K, Petersen KF, Shulman Gl. Molecular mechanisms of insulin resistance in humans and their potential links with mitochondrial dysfunction. Diabetes 2006;55(Suppl 2):S9-S15.

23. Befroy DE, Petersen KF, Dufour $S$ et al. Impaired mitochondrial substrate oxidation in muscle of insulin-resistant offspring of type 2 diabetic patients. Diabetes 2007;56:1376-1381.

24. Schrauwen P, Mensink M, Schaart G et al. Reduced skeletal muscle uncoupling protein- 3 content in prediabetic subjects and type 2 diabetic patients: restoration by rosiglitazone treatment. J Clin Endocrinol Metab 2006;91:1520-1525.

25. Koves TR, Ussher JR, Noland RC et al. Mitochondrial overload and incomplete fatty acid oxidation contribute to skeletal muscle insulin resistance. Cell Metab 2008;7:45-56.

26. Verdich C, Toubro S, Buemann B et al. Leptin levels are associated with fat oxidation and dietary-induced weight loss in obesity. Obes Res 2001;9:452-461.

27. Minokoshi Y, Kim YB, Peroni OD et al. Leptin stimulates fatty-acid oxidation by activating AMP-activated protein kinase. Nature 2002;415:339-343.

28. Turner N, Bruce CR, Beale SM et al. Excess lipid availability increases mitochondrial fatty acid oxidative capacity in muscle: evidence against a role for reduced fatty acid oxidation in lipid-induced insulin resistance in rodents. Diabetes 2007;56:2085-2092.

29. Schrauwen P, Hesselink MK, Blaak EE et al. Uncoupling protein 3 content is decreased in skeletal muscle of patients with type 2 diabetes. Diabetes 2001:50:2870-2873.

30. Schrauwen P, Hoeks J, Hesselink MK. Putative function and physiological relevance of the mitochondrial uncoupling protein-3: involvement in fatty acid metabolism? Prog Lipid Res 2006;45:17-41.

31. Nabben M, Hoeks J, Briedé JJ et al. The effect of UCP3 overexpression on mitochondrial ROS production in skeletal muscle of young versus aged mice. FEBS Lett 2008:582:4147-4152.

32. Dobbins RL, Szczepaniak LS, Bentley B et al. Prolonged inhibition of muscle carnitine palmitoyltransferase-1 promotes intramyocellular lipid accumulation and insulin resistance in rats. Diabetes 2001;50:123-130. 